\title{
Classical truth in higher types
}

\author{
Ulrich Berger*1 \\ ${ }^{1}$ Univ. of Wales Swansea, Dept. of Computer Science, Singleton Park, Swansea SA2 8PP, UK
}

\begin{abstract}
Key words Logic, higher types, constructive mathematics. MSC (2000) 03B15,03D65,03F10,03F50

We study, from a classical point of view, how the truth of a statement about higher type functionals depends on the underlying model. The models considered are the classical set-theoretic finite type hierarchy and the constructively more meaningful models of Continuous Functionals, Hereditarily Effective Operations, as well as the closed term model of Gödel's system $T$. The main results are characterisations of prenex classes for which truth in the full set-theoretic model transfers to truth in the other models. As a corollary we obtain that the axiom of choice is not conservative over Gödel's system $T$ with classical logic for closed $\exists^{2}$-formulas. We hope that this study will contribute to a clearer delineation and perception of constructive mathematics from a classical perspective.
\end{abstract}

Copyright line will be provided by the publisher

\section{Introduction}

In recent years a new branch of proof theory, dubbed "proof mining", has gained considerable popularity ([18], [16], [17], [1], [5], [15], [11], [3], [14], [2], [12] and many more). In proof mining one extracts computational content from (constructive and non-constructive) proofs in order to obtain formally verified, more efficient, or new algorithms, uniform bounds and effective rates of convergence. Very often the proofs are formulated in systems based on higher types and, most curiously, "false" axioms. That the extracted programs are nevertheless correct hinges on the fact that there are models of higher types where these "false" axioms are actually true (for continuity reasons, for example). An example of such an axiom is the principle of uniform $\Sigma_{1}^{0}$-boundedness stating that a type two functional restricted to a bounded (but nonetheless uncountable) set of arguments is bounded, uniformly in the bound of the argument set.

In this note we investigate in general how the truth of a statement involving higher type functionals depends on the underlying model. More precisely, given models $\mathcal{X}$ and $\mathcal{Y}$ of Gödel's system $T$ of primitive recursive functionals in higher types [8], it is our aim to characterise those higher type quantifier prefixes $\mathcal{Q}$ such that for all prenex formulas $A$ with quantifier prefix $\mathcal{Q}$ the truth of $A$ in $\mathcal{X}$ implies the truth of $A$ in $\mathcal{Y}$, in symbols: $\mathcal{X} \stackrel{\mathcal{Q}}{\Longrightarrow} \mathcal{Y}$. We will concentrate on the following four models of system $T$ : The full set-theoretic model $\mathbf{S}$, the model $\mathbf{C}$ of continuous functionals of Kleene and Kreisel [9, 13] (see also [20]), the model HEO of hereditarily effective operations and the closed term model $\mathbf{T}$ [20]. These models are of particular interest for proof mining, but they are also significant from a foundational point of view, since they correspond (roughly) to the classical, intuitionistic, constructivistic and formalistic perception of higher types. Our main result is that $\mathbf{S} \stackrel{\mathcal{Q}}{\Longrightarrow} \mathbf{C}$ holds if and only if $\mathcal{Q}$ contains no existential quantifier of a level higher than 1 (Theorem 4.9). The quantifier prefixes $\mathcal{Q}$ with $\mathbf{S} \stackrel{\mathcal{Q}}{\Longrightarrow}$ HEO and $\mathbf{S} \stackrel{\mathcal{Q}}{\Longrightarrow} \mathbf{T}$ are characterised in a similar way (Theorem 4.10). Technically, the core of this result is the construction of a Gödel primitive recursive functional of type 3 that has a zero, but no continuous zero (Lemma 4.2). As a corollary we obtain that the axiom of choice is not conservative over Gödel's system $T$ with classical logic for (closed) sentences of the form $\exists F^{2} . r=0$ (Corollary 4.4).

This note studies truth in classically and constructively meaningful models from a classical point of view. We hope that this contributes to a clearer delineation and better perception of constructive mathematics from the perspective of classical mathematics. A similar goal has, for example, undoubtedly been achieved through the (classical) characterisation of intuitionistic logic by Kripke models.

\footnotetext{
* Corresponding author: e-mail: u.berger@swansea.ac.uk, Phone: +00 441792 513380, Fax: +00 441792295708
} 


\section{Gödel's system $T$}

Types are the base type of natural numbers $\iota$ and function types $\rho \rightarrow \sigma$. Typ denotes the set of all types. As usual, $\rho_{1} \rightarrow \rho_{2} \rightarrow \ldots \rightarrow \rho_{n} \rightarrow \sigma$ stands for $\rho_{1} \rightarrow\left(\rho_{2} \rightarrow \ldots \rightarrow\left(\rho_{n} \rightarrow \sigma\right) \ldots\right)$. The level of of a type is defined as $\operatorname{lev}(\iota)=0, \operatorname{lev}(\rho \rightarrow \sigma)=\max (\operatorname{lev}(\rho)+1, \operatorname{lev}(\sigma))$. We use the numbers $0,1,2,3, \ldots$ as abbreviations for the types $\iota, \iota \rightarrow \iota,(\iota \rightarrow \iota) \rightarrow \iota,((\iota \rightarrow \iota) \rightarrow \iota) \rightarrow \iota, \ldots$, which are often called pure types. The level of the pure type $n$ is $n$.

Terms are built from typed variables $x^{\rho}, y^{\rho}, \ldots$ and the constant $0^{\iota}$ by the successor operation $\mathrm{S}\left(t^{\iota}\right)^{\iota}$, primitive recursion $\mathrm{R}_{\rho}\left(r^{\rho}, s^{\iota \rightarrow \rho \rightarrow \rho}\right)^{\iota \rightarrow \rho}$ ( $\rho \in$ Typ), $\lambda$-abstraction $\left(\lambda x^{\rho} . r^{\sigma}\right)^{\rho \rightarrow \sigma}$ and application $\left(r^{\rho \rightarrow \sigma} s^{\rho}\right)^{\sigma}$.

Formulas are built from equations between terms of type $\iota$ by the usual propositional connectives and the quantifiers $\forall x^{\rho}$ and $\exists x^{\rho}$ where $\rho$ is an arbitrary type.

The axioms of system $T$ are the usual $\beta$-equality $C\left[\left(\lambda x^{\rho} . r\right) s\right]=C\left[r\left[s / x^{\rho}\right]\right]$ and $\eta$-equality $C\left[\lambda x^{\rho} . r x\right]=C[r]$ ( $x$ not free in $r$ ) as well as the equations for primitive recursion at all types:

$$
\begin{aligned}
C\left[\mathrm{R}_{\rho}(r, s) 0\right] & =C[r] \\
C\left[\mathrm{R}_{\rho}(r, s) \mathrm{S}(t)\right] & =C\left[s t\left(\mathrm{R}_{\rho}(r, s) t\right)\right]
\end{aligned}
$$

where $C[$.$] is a term context of type \iota$ (with a hole "." of suitable type). We write $T \vdash A$ if the formula $A$ follows from the axioms of system $T$ by the usual rules of classical many-sorted predicate calculus (viewing types as sorts) and equality at type $\iota$. Hence we consider here a classical version of system $T$.

\section{Models}

We consider extensional models that interpret the base type $\iota$ in the standard way, but may vary in their interpretation of function types. More precisely, a pre-model is a family $\mathcal{X}=\left(\mathcal{X}_{\rho}\right)_{\rho \in \text { Typ }}$ where $\mathcal{X}_{\iota}$ is the set of natural numbers $\mathbf{N}=\{0,1,2, \ldots\}$ and $\mathcal{X}_{\rho \rightarrow \sigma} \subseteq \mathcal{X}_{\sigma}^{\mathcal{X}_{\rho}}$. A model of system $T$ is a pre-model $\mathcal{X}$ such that the value $\llbracket t^{\rho} \rrbracket \eta \in \mathcal{X}_{\rho}$ of a term $t^{\rho}$ with respect to an environment $\eta$, given by the equations below, is well-defined:

$$
\begin{aligned}
\llbracket x \rrbracket \eta & =\eta(x) \\
\llbracket 0 \rrbracket \eta & =0 \\
\llbracket \mathrm{S}(t) \rrbracket \eta & =\llbracket t \rrbracket+1 \\
\llbracket \mathrm{R}_{\rho}(r, s) \rrbracket \eta & =f: \mathbf{N} \rightarrow \mathcal{X}_{\rho} \text { where } f(0)=\llbracket r \rrbracket \eta, f(n+1)=(\llbracket s \rrbracket \eta)(n)(f(n)) \\
\llbracket \lambda x^{\rho} . r \rrbracket \eta & =g: \mathcal{X}_{\rho} \rightarrow \mathcal{X}_{\sigma} \text { where } g(a)=\llbracket r \rrbracket \eta[x:=a] \\
\llbracket r^{\rho \rightarrow \sigma} s^{\rho} \rrbracket \eta & =\llbracket r \rrbracket \eta(\llbracket s \rrbracket \eta)
\end{aligned}
$$

Note that the only possibility for the definition to fail is that the function $f$ above is not in $\mathcal{X}_{\iota \rightarrow \rho}$, or $g$ is not in $\mathcal{X}_{\rho \rightarrow \sigma}$. An element of a model is called Gödel primitive recursive if it can be defined by a closed term of system $T$. We write $\mathcal{X}, \eta \models A$ if the formula $A$ holds in the model $\mathcal{X}$ w.r.t. the environment $\eta$. If $A$ is closed we omit the environment and write $\mathcal{X} \models A$. It is easy to see that a model satisfies the axioms of system $T$. Therefore, $T \vdash A$ implies $\mathcal{X} \models A$ for every closed formula $A$. We consider the following four models:

1. The (standard) set-theoretic model $\mathbf{S}$, with $\mathbf{S}_{\rho \rightarrow \sigma}:=\mathbf{S}_{\sigma}^{\mathbf{S}_{\rho}}$.

2. The closed term model $\mathbf{T}=\left(\mathbf{T}_{\rho}\right)_{\rho \in \text { Typ }}$. $\mathbf{T}_{\rho}$ is defined by induction on $\rho$, simultaneously with relations $\sim_{\rho} \subseteq \operatorname{Ter}_{\rho} \times \mathbf{T}_{\rho}$ where $\operatorname{Ter}_{\rho}$ is the set of closed terms of type $\rho . t^{\iota} \sim_{\iota} n: \Leftrightarrow T \vdash t=n$ where on the right hand side $n$ means the $n$-th numeral. For a closed term $r^{\rho \rightarrow \sigma}$ and a function $f: \mathbf{T}_{\rho} \rightarrow \mathbf{T}_{\sigma}$ we define

$$
r \sim_{\rho \rightarrow \sigma} f: \Leftrightarrow \forall s \in \operatorname{Ter}_{\rho} \forall a \in \mathbf{T}_{\rho}\left(s \sim_{\rho} a \Rightarrow r s \sim_{\sigma} f(a)\right)
$$

and set $\mathbf{T}_{\rho \rightarrow \sigma}:=\left\{f \mid \exists r\left(r \sim_{\rho \rightarrow \sigma} f\right)\right\}$. The statement " $r \sim_{\rho} a$ " can be read as " $r$ represents (or realises) $a$ ".

3. The model HEO of hereditarily effective operations, which is defined similarly to 2., but with natural numbers and partial recursive function application instead of terms and term application [20].

4. The model $\mathbf{C}$ of continuous functionals, which we define following the same pattern as in 2 . and 3 . However the representing elements are now partial continuous functionals (following Ershov [4]), which are defined as follows: For every type $\rho$ we define a topological space $\hat{\mathbf{C}}_{\rho}$, the partial continuous functionals of type 
$\rho . \hat{\mathbf{C}}_{\iota}:=\mathbf{N} \cup\{\perp\}$ with the topology generated by the sets $\{n\}$ for $n \in \mathbf{N}$. The element $\perp$ represents the value "undefined". Note that the singleton set $\{\perp\}$ is not open. The only open set containing $\perp$ is the whole space. $\hat{\mathbf{C}}_{\rho \rightarrow \sigma}:=\left\{f: \hat{\mathbf{C}}_{\rho} \rightarrow \hat{\mathbf{C}}_{\sigma} \mid f\right.$ is continuous $\}$ endowed with the pointwise topology. Alternatively, the model $\hat{\mathbf{C}}$ can be constructed order-theoretically by defining an order on $\hat{\mathbf{C}}_{\iota}:=\mathbf{N} \cup\{\perp\}$ by $a \sqsubseteq b: \Leftrightarrow a \in\{\perp, b\}$ and setting $\hat{\mathbf{C}}_{\rho \rightarrow \sigma}:=\left\{f: \hat{\mathbf{C}}_{\rho} \rightarrow \hat{\mathbf{C}}_{\sigma} \mid f\right.$ is monotone and preserves directed suprema $\}$ endowed with the pointwise order. Each space $\hat{\mathbf{C}}_{\rho}$ is then a countable bounded complete algebraic domain in the sense of Scott's domain theory $[19,7]$. The two ways of defining $\hat{\mathbf{C}}$ are equivalent since the topology of the first definition is the Scotttopology of the domain ordering while the domain ordering can be defined from the topology as the specialisation order, i.e. $a \sqsubseteq b$ holds iff $a$ is in the (topological) closure of $\{b\}$. The model $\mathbf{C}=\left(\mathbf{C}_{\rho}\right)_{\rho \in \text { Typ }}$ together with representation relations $\sim_{\rho} \subseteq \hat{\mathbf{C}}_{\rho} \times \mathbf{C}_{\rho}$ is now defined in the expected way: $a \sim_{\iota} n: \Leftrightarrow a=n$ and for $f \in \hat{\mathbf{C}}_{\rho \rightarrow \sigma}$ and $g: \mathbf{C}_{\rho} \rightarrow \mathbf{C}_{\sigma}$ and

$$
f \sim_{\rho \rightarrow \sigma} g: \Leftrightarrow \forall a \in \hat{\mathbf{C}}_{\rho} \forall b \in \mathbf{C}_{\rho}\left(a \sim_{\rho} b \Rightarrow f(a) \sim_{\sigma} g(b)\right) .
$$

$\mathbf{C}_{\rho \rightarrow \sigma}:=\left\{g \mid \exists f\left(f \sim_{\rho \rightarrow \sigma} g\right)\right\}$.

Theorem 3.1 The pre-models $\mathbf{S}, \mathbf{C}, \mathbf{H E O}$ and $\mathbf{T}$ are models of system $T$.

The next theorem collects some well-known facts about these models. Items (b), (c) and (d) make use of the continuity predicate

$$
\operatorname{Cont}\left(F^{2}\right):=\forall g^{1} \exists k^{0} \forall h^{1}\left(g={ }_{k} h \rightarrow F(g)=F(h)\right)
$$

where $g={ }_{k} h$ means $\forall i<k(g(i)=h(i))$. Clearly, the ternary predicate $g={ }_{k} h$ can be encoded by an equation.

Theorem 3.2 (a) $\mathbf{T}_{1}=\varepsilon_{0}-\mathcal{R} \subset \mathbf{H E O}_{1}=\mathcal{R} \subset \mathbf{C}_{1}=\mathbf{S}_{1}=\mathbf{N}^{\mathbf{N}}$ where $\left(\varepsilon_{0^{-}}\right) \mathcal{R}$ is the set of $\left(\varepsilon_{0^{-}}\right)$recursive functions (see below).

(b) $\mathbf{C}_{2}=\left\{F \in \mathbf{S}_{2} \mid \operatorname{Cont}(F)\right\} \subset \mathbf{S}_{2}=\mathbf{N}^{\mathbf{S}_{1}}$, i.p. $\mathbf{C} \models \forall F^{2} \operatorname{Cont}(F)$.

(c) $\mathrm{HEO} \models \forall F^{2} \operatorname{Cont}(F)$.

(d) $\mathbf{T} \models \forall F^{2} \operatorname{Cont}(F)$.

Remark 3.3 Theorem 3.1 is folklore. Except for $\mathbf{S}$ all results are non-trivial. In Theorem 3.2, the first equation of part (a) follows from Gentzen's and Gödel's celebrated results that a computable function is definable in system $T$ iff its totality can be proven in Peano-Arithmetic [8], and this in turn is equivalent to the fact that it can be defined by a recursion along a wellordering less than $\varepsilon_{0}$ [6]. Part (b) is immediate from the definition of $\mathbf{C}_{2}$. Part (c) is a consequence of the Kreisel-Lacombe-Shoenfield-Theorem [10]. Part (d) follows fairly easily from the fact that $\mathbf{C}$ is a model of system $T$. Note that in (b) the variables $g$ and $h$ in the predicate $\operatorname{Cont}\left(F^{2}\right)$ range over all number-theoretic functions while in (c) they range over computable and in (d) over Gödel primitive recursive functions. See [20] for more information on these models.

\section{Comparing truth in higher types}

The goal of this paper is to compare the classical truth of formulas in different models. Because, classically, every formula is provably equivalent to a prenex formula it is natural to base the comparison on the latter. Let $\mathcal{Q}=Q_{1}^{\rho_{1}} \ldots Q_{n}^{\rho_{n}}$ where $Q_{i} \in\{\exists, \forall\}$ be a quantifier prefix. A $\mathcal{Q}$-formula is a formula $A$ that is provably equivalent to a prenex formula of the form $Q_{1} x_{1}^{\rho_{1}} \ldots Q_{n} x_{n}^{\rho_{n}}$. $A_{0}$ where $A_{0}$ is a quantifier-free formula. Since the boolean connectives can be represented by terms we may even assume that $A_{0}$ is an equation (between terms of base type).

Let $\mathcal{Q}$ be a quantifier prefix and $\mathcal{X}, \mathcal{Y}$ models of system $T$. We write $\mathcal{X} \stackrel{\mathcal{Q}}{\Longrightarrow} \mathcal{Y}$ if $\mathcal{X} \models A$ implies $\mathcal{Y} \models A$ for all closed $\mathcal{Q}$-formulas $A$. Note that $\mathcal{X} \stackrel{\mathcal{Q}}{\Longrightarrow} \mathcal{Y}$ iff $\mathcal{Y} \stackrel{\overline{\mathcal{Q}}}{\Longrightarrow} \mathcal{X}$ where $\overline{\mathcal{Q}}$ is the dual prefix obtained by swapping $\forall$ and $\exists$.

By $\mathcal{X} \stackrel{?}{\Longrightarrow} \mathcal{Y}$ we denote the problem of determining the quantifier prefixes $\mathcal{Q}$ for which $\mathcal{X} \stackrel{\mathcal{Q}}{\Longrightarrow} \mathcal{Y}$ holds. By the remark above the problems $\mathcal{X} \stackrel{?}{\Longrightarrow} \mathcal{Y}$ and $\mathcal{Y} \stackrel{?}{\Longrightarrow} \mathcal{X}$ are equivalent. In this note we solve the problems 
$\mathbf{S} \stackrel{?}{\Longrightarrow} \mathbf{C}, \mathbf{S} \stackrel{?}{\Longrightarrow}$ HEO and $\mathbf{S} \stackrel{?}{\Longrightarrow} \mathbf{T}$. In order to develop some intuition for the relation $\mathcal{X} \stackrel{\mathcal{Q}}{\Longrightarrow} \mathcal{Y}$ we discuss an example:

Example 4.1 Consider the type 2 functional $E^{2}: \mathbf{N}^{\mathbf{N}} \rightarrow \mathbf{N}$ defined by $E(f)=1$ if $f(k)>0$ for some $k \in$ $\mathbf{N}$, and $E(f)=0$ if $f$ is constant 0 . Clearly, $E$ is non-continuous. This shows that the formula $\exists F^{2} \neg \operatorname{Cont}(F)$ is true in $\mathbf{S}$ while, according to Theorem 3.2 (b), it is false in $\mathbf{C}$. Therefore, since $\exists F^{2} \neg \operatorname{Cont}(F)$ is an $\exists^{2} \exists^{1} \forall^{0} \exists^{1}$ formula, $\mathbf{S} \stackrel{\exists^{2} \exists^{1} \forall^{0} \exists^{1}}{\Longrightarrow} \mathbf{C}$ does not hold. Using standard coding techniques we can contract the first two quantifiers and obtain that even $\mathbf{S} \stackrel{\exists^{2} \forall^{0} \exists^{1}}{\Longrightarrow} \mathbf{C}$ fails. But we can do better. The functional $E$ satisfies $E(\lambda x .0)=0$ and $E(\lambda n . n>k)=1$ for all $k$, where $n>k$ is 1 if $n$ is greater than $k$ and 0 otherwise. Furthermore, these facts alone imply that $E$ is non-continuous. Hence the $\exists^{2} \forall^{0}$-formula

$$
\exists F^{2}(F(\lambda x .0)=0 \wedge \forall k(F(\lambda n . k<n)=1))
$$

holds in $\mathbf{S}$, but not in $\mathbf{C}$, showing that $\mathbf{S} \stackrel{\exists^{2} \forall^{0}}{\Longrightarrow} \mathbf{C}$ fails.

The example above raises the question whether $\mathbf{S} \stackrel{\Xi^{2}}{\Longrightarrow} \mathbf{C}$ fails as well? At first glance this seems unlikely since any satisfiable property of type 2 functionals forcing non-continuity seems to require a universal quantification over infinitely many natural numbers, because, roughly, continuity is about what happens at infinity. However, we have the following:

Lemma 4.2 There exists a Gödel primitive recursive functional in $\mathbf{S}_{3}$ that has a zero, but no continuous zero.

Proof. Define $\Phi: \mathbf{S}_{2} \rightarrow \mathbf{N}$ by

$$
\Phi\left(F^{2}\right)=\left(1 \dot{-} F\left(\lambda x^{0} .1\right)\right)+F\left(\lambda x^{0} .1 \dot{-} \Pi_{y \leq x} F(\lambda z . z>y)\right)
$$

where - is the usual "cut-off" subtraction, and $\prod_{y \leq x} f(y)=f(0) * f(1) \ldots * f(x)$ with $*$ denoting multiplication. Clearly, $\Phi$ is Gödel primitive recursive.

We first show that $\Phi(E)=0$, where $E$ is the functional defined in Example 4.1. Since $E(\lambda x .1)=1$ we have $1-E(\lambda x .1)=0$. Furthermore, $E(\lambda z . z>y)=1$ for every $y \in \mathbf{N}$. Hence $\Pi_{y \leq x} E(\lambda z . z>y)=1$ for every $x \in \mathbf{N}$ and consequently $\lambda x .1-\Pi_{y \leq x} E(\lambda z . z>y)$ is the constant 0 function which is mapped to 0 by $E$. This shows that $\Phi(E)=0$.

Now let $F \in \mathbf{S}_{2}$ be such that $\Phi(F)=0$, i.e.

(1) $F(\lambda x .1)>0$,

(2) $F\left(\lambda x^{0} .1-\Pi_{y \leq x} F(\lambda z . z>y)\right)=0$.

We need to show that $F$ is not continuous. Clearly, it suffices to show

(*) $F(\lambda z . z \geq n)>0$ for all $n \in \mathbf{N}$.

(**) $F(\lambda x .0)=0$.

We prove (*) by induction on $n$. Base. Since $\lambda z . z \geq 0=\lambda z .1$ we have $F(\lambda z . z \geq 0)>0$, by (1). Step. Assume, for contradiction, $F(\lambda z . z \geq(n+1))=0$, i.e. $F(\lambda z . z>n)=0$. We show that $1-\Pi_{y \leq x} F(\lambda z . z>y)=x \geq n$ for all $x$. If $x<n$, then for all $y \leq x$ we have $F(\lambda z . z>y)=F(\lambda z . z \geq y+1)>0$, by induction hypothesis. Hence $1-\Pi_{y \leq x} F(\lambda z . z>y)=0=(x \geq n)$. If, on the other hand, $x \geq n$, then $\Pi_{y \leq x} F(\lambda z . z>y)=0$ since, by (contradiction-) assumption, $F(\lambda z . z>n)=0$. Hence $1-\Pi_{y \leq x} F(\lambda z . z>y)=1=(x \geq n)$. Since we have shown $\lambda x .1-\Pi_{y \leq x} F(\lambda z . z>y)=\lambda x . x \geq n$ it follows by (2) that $F(\lambda x . x \geq n)=0$, contradicting the induction hypothesis. This ends the inductive proof of $(*)$.

Proof of (**): From (*) it follows that $\lambda x .1 \dot{-} \Pi_{y \leq x} F(\lambda z . z>y)=\lambda x .0$, hence $F(\lambda x .0)=0$, by (2). This completes the proof of $(* *)$ and of the lemma.

$$
\text { Corollary } 4.3 \mathrm{~S} \stackrel{\exists^{2}}{\Longrightarrow} \mathrm{C} \text { fails. }
$$


Pro of. Let $t^{3}$ be the closed term defining the functional $\Phi$ of Lemma 4.2 and $A$ the $\exists^{2}$-formula $\exists F^{2}(t F=$ 0 ). Then, according to Lemma 4.2, $A$ holds in $\mathbf{S}$, but not in $\mathbf{C}$.

Corollary 4.4 The axiom of choice is not conservative over (the classical version of) Gödel's system $T$ for closed $\exists^{2}$-formulas.

Proof. Let $A$ be as in the proof of Corollary 4.3. The proof of Lemma 4.2 shows that $A$ is provable with classical logic and the axiom of choice (more precisely an instance of the axiom of choice of the form $\forall f^{1} \exists x^{0} B(f, x) \rightarrow \exists F^{2} \forall f^{1} B(f, F(f))$ where $B$ is a $\forall^{0}$-formula, which suffices to show the existence of $E^{2}$ ). However, the formula $A$ cannot be provable in classical system $T$ since by Lemma 4.2 and Theorem 3.2 (b), $A$ is false in $\mathbf{C}$, which, by Theorem 3.1, is a (classical) model of system $T$.

Remark 4.5 Conservativity does hold for closed $\exists^{1}$-formulas, since if classical logic plus the axiom of choice proves $\exists f^{1} \cdot t^{2} f=0$ ( $t$ closed), then this formula holds in $\mathbf{S}$. Furthermore, it follows from Lemma 4.7 below that $t$ has the same value in $\mathbf{S}$ and $\mathbf{C}$, and hence $\llbracket t \rrbracket \in \mathbf{C}_{2} \subset \mathbf{S}_{2}$ is continuous. Therefore, there exists a closed term $s^{1}$ (defining a function which is 0 almost everywhere) such that $\llbracket t s \rrbracket=0$ and therefore $t s=0$ is provable by purely equational reasoning (since system $T$ is normalising). Hence $\exists f^{1}$.t $f=0$ is provable in system $T$ (even in its intuitionistic version).

We now draw our attention to the problem of proving that a statement of the form $\mathcal{X} \stackrel{\mathcal{Q}}{\Longrightarrow} \mathcal{Y}$ holds. The key idea is to relate the models $\mathcal{X}$ and $\mathcal{Y}$ via a logical relation similar to the relations used to define the models $\mathbf{T}$, $\mathbf{H E O}$ and $\mathbf{C}$.

Let $\mathcal{X}$ and $\mathcal{Y}$ be pre-models. Define for every type $\rho$ a relation $\sim_{\rho} \subseteq \mathcal{X}_{\rho} \times \mathcal{Y}_{\rho}$ by $x \sim_{\iota} y: \Leftrightarrow x=y$ and

$$
f \sim_{\rho \rightarrow \sigma} g: \Leftrightarrow \forall a \in \mathcal{X}_{\rho} \forall b \in \mathcal{Y}_{\rho}\left(a \sim_{\rho} b \Rightarrow f(a) \sim_{\sigma} g(b)\right)
$$

Lemma 4.6 Let $\mathcal{Y}$ be a pre-model. Then for every type $\rho$ we have:

(a) For every $b \in \mathcal{Y}_{\rho}$ there exists at least one $a \in \mathbf{S}_{\rho}$ such that $a \sim_{\rho} b$.

(b) For every $a \in \mathbf{S}_{\rho}$ there exists at most one $b \in \mathcal{Y}_{\rho}$ such that $a \sim_{\rho} b$.

Proof. We prove (a) and (b) simultaneously by induction on $\rho$. The base case is trivial.

Step, $\rho \rightarrow \sigma$. (a): Let $g \in \mathcal{Y}_{\rho \rightarrow \sigma}$. We define $f \in \mathbf{S}_{\rho \rightarrow \sigma}$ as follows. Let $a \in \mathbf{S}_{\rho}$. If there exists some $b \in \mathcal{Y}_{\rho}$ such that $a \sim_{\rho} b$, then this $b$ is unique, by induction hypothesis (b). By induction hypothesis (a), we have $c \sim_{\sigma} g(b)$ for some $c \in \mathbf{S}_{\sigma}$. Set $f(a):=c$. If such $b$ does not exist, then we define $f(a)$ as an arbitrary element of $\mathbf{S}_{\sigma}$. We need to show $f \sim_{\rho \rightarrow \sigma} g$. If $a \sim_{\rho} b^{\prime}$, then we are in the first case of the definition of $f$ and $b^{\prime}=b$ by uniqueness. Hence $f(a)=c \sim_{\sigma} g(b)=g\left(b^{\prime}\right)$.

(b): Assume $f \sim_{\rho \rightarrow \sigma} g$ and $f \sim_{\rho \rightarrow \sigma} g^{\prime}$. We need to show $g=g^{\prime}$. Let $b \in \mathcal{Y}_{\rho}$. By induction hypothesis (a) there exists $a \in \mathbf{S}_{\rho}$ such that $a \sim_{\rho} b$. It follows $f(a) \sim_{\sigma} g(b)$ and $f(a) \sim_{\sigma} g^{\prime}(b)$. By induction hypothesis (b) we have $g(b)=g^{\prime}(b)$.

If $\mathcal{X}, \mathcal{Y}$ are pre-models and $\eta, \xi$ environments for $\mathcal{X}, \mathcal{Y}$, then we write $\eta \sim \xi$ to mean that $\eta\left(x^{\sigma}\right) \sim_{\sigma} \xi\left(x^{\sigma}\right)$ for every variable $x^{\sigma}$.

Lemma 4.7 (Fundamental lemma for logical relations) Let $\mathcal{X}, \mathcal{Y}$ be models of system $T$ and $\eta, \xi$ environments for $\mathcal{X}, \mathcal{Y}$ such that $\eta \sim \xi$. Then $\llbracket r \rrbracket^{\mathcal{X}} \eta \sim_{\rho} \llbracket r \rrbracket^{\mathcal{Y}} \xi$ for every term $r^{\rho}$ of system $T$.

Pro of. Easy induction on $r$, using a side induction on natural numbers in the case where $r$ is of the form $\mathrm{R}_{\rho}(r, s)$.

Lemma 4.8 Let $\eta$, $\xi$ be environments for $\mathbf{S}$ and $\mathbf{C}$ such that $\eta \sim \xi$. Let $\mathcal{Q}$ be a quantifier prefix such that every existential quantifier in $\mathcal{Q}$ has type level $\leq 1$. Then $\mathbf{S}, \eta=A$ implies $\mathbf{C}, \xi=A$ for every $\mathcal{Q}$-formula $A$. 
Proof. We may assume that $A$ is of the form $Q_{1} x_{1}^{\rho_{1}} \ldots Q_{n} x_{n}^{\rho_{n}} \cdot r^{\iota}=s^{\iota}$ where $\mathcal{Q}=Q_{1}^{\rho_{1}} \ldots Q_{n}^{\rho_{n}}$ and $\operatorname{lev}\left(\rho_{i}\right) \leq 1$ if $Q_{i}=\exists$. We proceed by induction on formulas of that shape.

$r^{\iota}=s^{\iota}$ : Assume $\mathbf{S}, \eta \models r=s$, i.e. $\llbracket r \rrbracket^{\mathbf{S}} \eta=\llbracket s \rrbracket^{\mathbf{S}} \eta$. By Lemma 4.7 we have $\llbracket r \rrbracket^{\mathbf{S}} \eta=\llbracket r \rrbracket^{\mathbf{C}} \xi$ and $\llbracket s \rrbracket^{\mathbf{S}} \eta=$ $\llbracket s \rrbracket^{\mathbf{C}} \xi$, since $\sim_{\iota}$ is the equality relation on $\mathbf{N}$. Therefore $\mathbf{C}, \xi \models r=s$.

$\forall x^{\rho} A$ : Assume $\mathbf{S}, \eta \models \forall x^{\rho} A$, i.e. $\mathbf{S}, \eta[x:=a] \models A$, for all $a \in \mathbf{S}_{\rho}$. Let $b \in \mathbf{C}_{\rho}$. By Lemma 4.6 (a) there exists some $a \in \mathbf{S}_{\rho}$ with $a \sim_{\rho} b$. Hence $\eta[x:=a] \sim \xi[x:=b]$. By induction hypothesis, $\mathbf{C}, \xi[x:=b] \models A$.

$\exists x^{\rho} A$ where $\operatorname{lev} \rho \leq 1$ : Assume $\mathbf{S}, \eta \models \exists x^{\rho} A$, i.e. $\mathbf{S}, \eta[x:=a] \models A$, for some $a \in \mathbf{S}_{\rho}$. Since $\operatorname{lev}(\rho) \leq 1$ we have $\mathbf{S}_{\rho}=\mathbf{C}_{\rho}$ (clear, by the definition of $\mathbf{C}$ ). Furthermore $a \sim_{\rho} a$. Hence, $\eta[x:=a] \sim \xi[x:=a]$ and consequently $\mathbf{C}, \xi[x:=a] \models A$, by induction hypothesis.

Theorem 4.9 $\mathbf{S} \stackrel{\mathcal{Q}}{\Longrightarrow} \mathbf{C}$ iff every existential quantifier in $\mathcal{Q}$ has type level $\leq 1$.

Pro of. The "only if" direction follows from Corollary 4.3. The "if" direction follows from Lemma 4.8.

Theorem 4.10 Let $\mathcal{X}$ be a model of system $T$ such that $\mathcal{X} \models \forall F^{2} \operatorname{Cont}(F)$ and such that all functions in $\mathcal{X}_{1}$ are computable (for example, $\mathcal{X}=\mathbf{H E O}$ or $\mathcal{X}=\mathbf{T}$ ). Then $\mathbf{S} \stackrel{\mathcal{Q}}{\Longrightarrow} \mathcal{X}$ iff every existential quantifier in $\mathcal{Q}$ has type level $\leq 1$ and no existential quantifier of level 1 in $\mathcal{Q}$ has a universal quantifier further to the right.

Proof. The "only if" direction follows from Lemma 4.2 and the fact that if $R$ is a binary primitive recursive relation on natural numbers such that $\{n \mid \exists k R(n, k)\}$ is undecidable, then there is no computable $f$ with $\forall n \forall k(R(n, k) \rightarrow R(n, f(n)))$. The "if" direction can be proved similarly to Lemma 4.8 using in the case $\exists f^{1} A$, where $A$ is quantifier free, an argument similar to the remark following Corollary 4.4.

\section{References}

[1] F. Barbanera and S. Berardi. Extracting constructive content from classical logic via control-like reductions. In M. Bezem and J.F. Groote, editors, Typed Lambda Calculi and Applications, pages 45-59. LNCS Vol. 664, 1993.

[2] A. Beckmann. Dynamic ordinal analysis. Archive for Mathematical Logic, 42:303-334, 2003.

[3] U. Berger, H. Schwichtenberg, and M. Seisenberger. The Warshall algorithm and Dickson's lemma: Two examples of realistic program extraction. Journal of Automated Reasoning, 26(2), 2001.

[4] Y.L. Ershov. Model $C$ of partial continuous functionals. In R. Gandy and M. Hyland, editors, Logic Colloquium 1976, pages 455-467. North-Holland, 1977.

[5] F. Ferreira. A feasible theory for analysis. Jour. Symb. Logic, pages 1001-1011, 1994.

[6] G. Gentzen. Beweisbarkeit und Unbeweisbarkeit von Anfangsfällen der transfiniten Induktion in der reinen Zahlentheorie. Mathematische Annalen, 119:140-161, 1943.

[7] G. Gierz, K.H. Hofmann, K. Keimel, J.D. Lawson, M. Mislove, and D.S. Scott. Continuous Lattices and Domains, volume 93 of Encyclopedia of Mathematics and its Applications. Cambridge University Press, 2003.

[8] K. Gödel. Über eine bisher noch nicht benützte Erweiterung des finiten Standpunktes. Dialectica, 12:280-287, 1958.

[9] S. C. Kleene. Countable functionals. In A. Heyting, editor, Constructivity in Mathematics, pages 81-100. NorthHolland, 1959.

[10] G. Kreisel, D. Lacombe, and J.R. Shoenfield. Partial Recursive Functionals and Effective Operations. Constructivity in Mathematics, pages 290-297, 1959.

[11] U. Kohlenbach. Mathematically strong subsystems of analysis with low rate of growth of provably recursive functionals. archive, 36:31-71, 1996.

[12] U. Kohlenbach. Proof mining in $L_{1}$-approximation. Annals of Pure and Applied Logic, 121:1-38, 2003.

[13] G. Kreisel. Interpretation of analysis by means of constructive functionals of finite types. Constructivity in Mathematics, pages 101-128, 1959.

[14] P. Letouzey. A New Extraction for Coq. In H. Geuvers and F. Wiedijk, editors, Types for Proofs and Programs, Second International Workshop, TYPES 2002, volume 2646 of LNCS. Springer-Verlag, 2003.

[15] H. Luckhardt. Bounds extracted by Kreisel from ineffective proofs. In P. Odifreddi, editor, Kreiseliana: About and around George Kreisel, pages 289-300. A. K. Peters, Wellesley, MA, 1996.

[16] C. Murthy. Extracting Constructive Content from Classical Proofs. PhD thesis, Cornell University, 1990.

[17] M. Parigot. $\lambda \mu$-calculus: an algorithmic interpretation of classical natural deduction. In Proceedings of Logic Programming and Automatic Reasoning, St. Petersburg, volume 624 of LNCS, pages 190-201. Springer, 1992.

[18] C. Paulin-Mohring. Extracting $F_{\omega}$ 's programs from proofs in the Calculus of Constructions. In Sixteenth Annual ACM Symposium on Principles of Programming Languages, Austin, January 1989. ACM.

[19] D. S. Scott. Outline of a mathematical theory of computation. In 4th Annual Princeton Conference on Information Sciences and Systems, pages 169-176, 1970.

[20] A.S. Troelstra. Metamathematical Investigation of Intuitionistic Arithmetic and Analysis, volume 344 of LNM. Springer, 1973. 